\title{
Orthotopic Heart Transplantation following univentricular palliation: new challenges for the congenital cardiac surgeon
}

\author{
María-Teresa González-López*, Juan-Miguel Gil-Jaurena', Ramón Pérez-Caballero-Martínez', \\ Ana-María Pita-Fernández ${ }^{1}$, Manuela Camino-López², Nuria Gil-Villanueva², José-Luis Zunzunegui-Martínez², \\ Constancio Medrano-López ${ }^{2}$
}

From World Society of Cardiothoracic Surgeons 25th Anniversary Congress, Edinburgh Edinburgh, UK. 19-22 September 2015

\section{Background/Introduction}

Orthotopic heart transplantation after univentricular palliation presents a difficult challenge due to the complex anatomy and prior surgeries.

\section{Aims/Objectives}

We present our surgical techniques/results in the current era.

\section{Method}

2013-2014: 23 congenital cardiac patients underwent heart transplantation. 13 of them: previous univentricular palliation: hypoplastic left heart syndrome $(n=8)$, dtransposition of great arteries+criss-cross-heart $(n=1)$, unbalanced atrioventricular septal defect $(n=1)$, pulmonary atresia+intact ventricular septum $(\mathrm{n}=1)$ and grown-up patients $(\mathrm{GUCH})(\mathrm{n}=2: 1$ double-inlet-leftventricle and 1 tricuspid atresia).

\section{Results}

Paediatric group $(\mathrm{n}=11)$ : age $7.1+/-4.8$ years (range 1.5 months-13 years); weight 21.3+/-9.5 kilograms (range 3.5-36). GUCH-group $(\mathrm{n}=2)$ : age $23.5+/-0.5$ years; weight $50+/-10.2$ kilograms. $46.1 \%$ had undergone Fontan completion, 15.4\% Fontan take-down, 30.7\% bidirectional cavopulmonary shunt and $7.7 \%$ Blalock-Taussig shunt. BerlinHeart-EXCOR-Paediatric-Device as bridge to transplantation was used in 1 patient. Bicaval technique was performed along with: hemiarch repair $(15.3 \%, \mathrm{n}=2)$, pulmonary artery

Paediatric Cardiac Surgery Department, Gregorio Marañón Hospital, Madrid, Spain

Full list of author information is available at the end of the article
(PA) branches plasty $(38.4 \%, \mathrm{n}=5)$, hilum-to-hilum PA reconstruction $(53.8 \%, \mathrm{n}=7)$, superior venae cavae reconstruction $(15.4 \%, \mathrm{n}=2)$ and stent removal from PA $(61.5 \%$, $\mathrm{n}=8)$, inferior venae cavae $(7.7 \%, \mathrm{n}=1)$ and lateral-tunnelFontan $(7.7 \%, \mathrm{n}=1)$. Average cardiopulmonary-bypass time 257.6+/-79.3 minutes (range 120-431); total-ischemia-time 220.7+/-48.6 (range 140-287). One patient required ECMO; 4 underwent delayed sternal closure; 2 underwent diaphragm plication; 1 subacute-humoral-rejection treated with plasmapheresis. In-hospital stay $44+/-16$ days (range 18-185). At follow-up (14.4+/-7.2 months), freedom from percutaneous procedures $83.3 \%(\mathrm{n}=10)$. 30-day mortality/ follow-up mortality: zero. All of them remain with an optimal functional class.

\section{Discussion/Conclusion}

Heart transplantation following univentricular palliation is technically demanding but short-term results are excellent. An extensive surgical reconstruction (donor/heterologous tissues) is mandatory to improve outcomes. Further follow-up is necessary to evaluate the long-term results in this scenario.

\section{Authors' details \\ 'Paediatric Cardiac Surgery Department, Gregorio Marañón Hospital, Madrid, Spain. ${ }^{2}$ Paediatric Cardiology Department, Gregorio Marañón Hospital, Madrid, Spain.}

Published: 16 December 2015

\section{doi:10.1186/1749-8090-10-S1-A92}

Cite this article as: González-López et al:: Orthotopic Heart

Transplantation following univentricular palliation: new challenges for the congenital cardiac surgeon. Journal of Cardiothoracic Surgery 2015 10(Suppl 1):A92. 